\title{
MINOS Experiment at Fermilab
}

\author{
T. Kafka, ${ }^{1}$ \\ for the MINOS Collaboration \\ ${ }^{1}$ Tufts University, Medford, MA, USA
}

November 20, 2009

\begin{abstract}
MINOS is a two-detector experiment to study neutrino oscillations in the NuMI high-intensity neutrino beam at the Fermi National Accelerator Laboratory. Results on $\nu_{\mu}$ disappearance, $\nu_{e}$ appearance, sterile neutrino mixing ( $\nu$ neutral current 'disappearance'), and $\bar{\nu}_{\mu}$ disappearance are summarized for an exposure of $\approx 3 \times 10^{20}$ protons on target. Data from a $7 \times 10^{20}$ POT exposure already in hand are being analyzed.
\end{abstract}

\section{Introduction}

MINOS (Main Injector Neutrino Ooscillation Search) is a long-baseline experiment running at Fermilab since 2005 in the intense NuMI (Neutrinos at the Main Injector) beam. The 1-kton Near Detector (ND) is located $1 \mathrm{~km}$ from the target at Fermilab, and the 5.4 kton Far Detector is located $735 \mathrm{~km}$ away, $700 \mathrm{~m}$ underground, in the Soudan Underground Mine State Park in Soudan, Minnesota. Both detectors constist of 2.54-cm thick steel planes interleaved with solid-scintillator planes composed of scintillator strips with a rectangular cross-section of $4.1 \times 1 \mathrm{~cm}^{2}$. Each strip has a wavelength-shifting optical fiber embedded that is read out by a multianode photomultiplier tube. Both detectors are magnetized to 1.3 T.[1]

All results reported here were obtained using the methodology of blind analysis.

In addition to studying oscillations, the large-statistics data sample of $\nu$ interactions in the Near Detector is being analyzed for various aspects of non-oscillation neutrino physics.

\section{$2 \quad \nu_{\mu}$ Disappearance}

A multivariate ' $k$-nearest-neighbors' algorithm was used to separate $\nu_{\mu}$ Charged-Current (CC, finalstate muon track present) and Neutral-Currrent (NC, final-state muon track absent) interactions for this analysis. The resulting CC sample had an NC background of only $0.6 \%$.

As the neutrino energy, $E_{\nu}$, distributions differ by $20 \%$ due to meson-decay kinematics, beamline geometry and detector acceptance, care has to be taken when extrapolating the ND spectrum to the FD to provide the expected 'no-oscillations' spectrum. For the CC analysis, the differences between ND and FD distributions were encoded into a Beam Transfer Matrix using Monte Carlo simulation.

The $\nu_{\mu}$ CC data set included both Low-Energy (LE) and High-Energy (HE) beam data from an exposure of $3.36 \times 10^{20}$ Protons On Target (POT). MINOS observed 848 events in $3.36 \times 10^{20}$ POT, 
while $1065 \pm 60$ events were expected with no oscillations. The $\nu_{\mu} \mathrm{CC}$ disappearance was confined to $E_{\nu}<10 \mathrm{GeV}$. When analyzed within the framework of two-neutrino mixing, $\nu_{\mu} \rightarrow \nu_{\tau}$, the MINOS data yielded the following limits on the mixing parameters: [2] $\left|\Delta m^{2}\right|=(2.43 \pm 0.13) \times 10^{-3} \mathrm{eV}^{2}$ at the $68 \%$ C.L., and $\sin ^{2}(2 \theta)>0.95$ at the $68 \%$ C.L. (and $>0.90$ at $90 \%$ C.L.). The fit had $\chi^{2} / N_{D o F}=90 / 97$.

The same data disfavor a pure decay as a reason for the $\nu_{\mu}$ disapppearance at $3.7 \sigma$. (It is $5.4 \sigma$ when $\mathrm{CC}$ and $\mathrm{NC}$ data are combined.) Pure decoherence is disfavored by the CC data at $5.7 \sigma$.

\section{$3 \quad \nu_{e}$ Appearance}

Search for $\nu_{e}$ appearance represents an attempt to find a small signal in the presence of a large background. The MINOS $\nu_{e}$ analysis group developed the following strategy for the task: (i) Select $\nu_{e} \mathrm{CC}$ candidate events in both MINOS detectors; (ii) Selected ' $\nu_{e}$ ' events in the Near Detector are all background - misidentified NC events, misidentified high- $y \nu_{\mu} \mathrm{CC}$ events, and intrinsic beam $\nu_{e}$ interactions; (iii) Extrapolate the number of background events to the Far Detector taking into account $\nu_{\mu} \rightarrow \nu_{\tau}$ oscillation for the $\nu_{\mu}$ CC background; (iv) Look for an excess of $\nu_{e}$ CC events in the FD data.

Event selection, including preliminary cuts (track length $<25$ planes, $1<E_{\nu}<8 \mathrm{GeV}$, shower present) and an applicaton of the Artificial Neural Network algorithm, leads to reduction of the Signalto-Background ratio from 1:55 to 1:4. To measure the composition of the background, MINOS is using a data-based method utilizing different $\mathrm{NC}$ and $\mathrm{CC}$ content in the Horn-on and Horn-off beam configuration. The resulting composition was found to be $(57 \pm 5) \% \mathrm{NC},(32 \pm 7) \% \mathrm{CC}$, and $(11 \pm 3) \%$ beam $\nu_{e}$ at the Near Detector. This background was propagated from the ND to the FD yielding an expected background in the Far Detector of $27 \pm 5$ (stat) \pm 2 (sys) for an exposure of $3.14 \times 10^{20}$ POT. $^{1}$

The signal determination algorithm was established prior to "Blinded Box" opening by maximizing

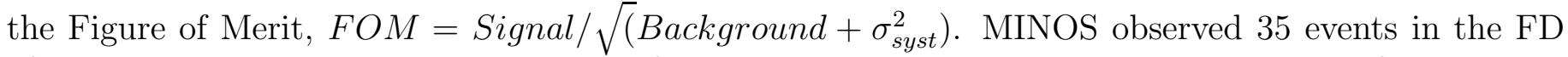
after selection, representing an 'excess' of $1.5 \sigma$ over the expected background. When fitted to the $\nu_{\mu} \rightarrow \nu_{e}$ oscillation hypothesis, this excess yields $90 \%$ C.L. contours in $\delta_{C P}$ vs. $\sin ^{2}\left(2 \theta_{13}\right)$ depicted in Fig. 1 for both normal and inverted neutrino mass hierarchy.

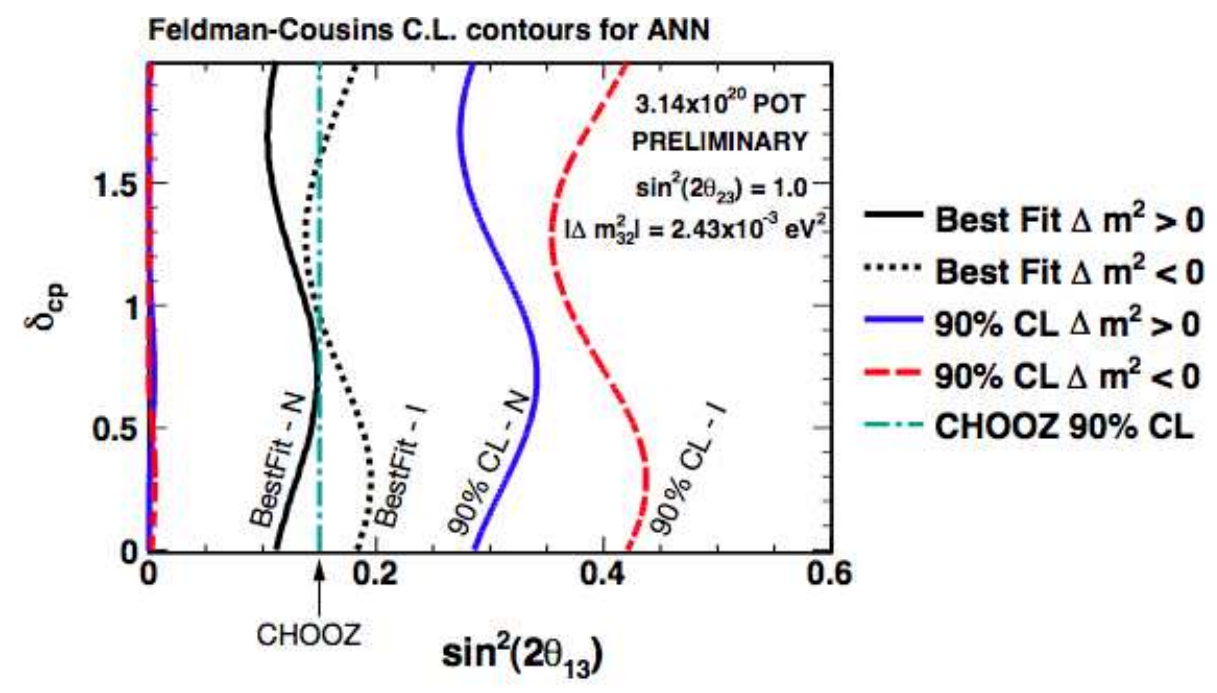

Figure 1: MINOS $\nu_{e}$ appearance contours: 'N' = Normal Hierarchy, 'I' = Inverted Hierarchy.

\footnotetext{
${ }^{1}$ Alternate algorithms for event selection and bckground decomposition yielded values compatible with the results given here.
} 


\section{Neutral-Current disappearance and $\nu_{s}$ mixing}

The MINOS NC analysis group looked for a dearth of NC neutrino interactions at the FD as a possible indication of sterile neutrino mixing. As the $\mathrm{NC}$ interaction rates are the same for all active $\nu$ flavors, the oscillations among the active flavors do not affect the $\mathrm{NC}$ spectrum. The $\nu_{s}$ would not interact in the detector, hence the $\nu_{s}$ footprint would be an energy-dependent depletion of the NC spectrum at the FD.

The analysis is cut based, and any remaining $\mathrm{CC}$ background is straightforward to estimate. The final NC sample was selected with an efficiency of $90 \%$ and purity of $60 \%$. Extrapolation from ND to FD used the "Far/Near" method, $F D_{i}^{\text {predicted }}=\left(F D_{i}^{M C} / N D_{i}^{M C}\right) N D_{i}^{\text {data }}$ in bins of $E_{\nu}$.

For a beam exposure of $3.18 \times 10^{20}$ POT, MINOS observes 388 events in the FD, when $377 \pm 19$ (stat) \pm 18 (syst) events were expected. indicating that the data is consistent with no NC disappearance. (This result updates report [3] describing a $2.46 \times 10^{20}$ POT data set.)

\section{$5 \quad \bar{\nu}_{\mu}$ Disappearance}

In the NuMI beam running in the "Forward-Horn-Current" LE configuration, 91.7\% of the beam neutrinos are $\nu_{\mu}$, however, the beam also contains $7.0 \% \bar{\nu}_{\mu}$, the remainder being electron flavored $\nu$. The $\nu_{\mu}$ energy distribution is optmized for studying oscillations (peak at $3 \mathrm{GeV}$ ), while the $\bar{\nu}_{\mu}$ energies are higher, with a peak at $8 \mathrm{GeV}$ - not optimal for measuring $\bar{\nu}_{\mu}$ oscillation, that is. But MINOS tried anyway.

The $\bar{\nu}_{\mu}$ analysis group devised an event selection suitable to minimize the background of misidentified $\nu_{\mu} \mathrm{CC}$ events $\left(\mu^{-}\right.$misidentified as $\left.\mu+\right)$ and NC events $\left(\pi^{+}\right.$misidentified as $\left.\mu^{+}\right)$. This selection had an efficiency of $80 \%$ and purity of $95 \%$. The selected data were then extrapolated from ND to FD via the Beam Transfer Matrix method. The MINOS $\bar{\nu}_{\mu}$ data sample at the FD had 42 events for a LE beam exposure of $3.2 \times 10^{20} \mathrm{POT}$. The prediction was $64.6 \pm 8.0$ (stat) \pm 3.0 (syst) events in the case of null oscillations, and 58.3 \pm 7.6 (stat) \pm 3.6 (syst) events for oscillation parameters measured by MINOS for $\nu_{\mu}$ oscilations.

This represents the first direct observation of $\bar{\nu}_{\mu}$ disappearance of an accelerator long-baseline experiment. The difference between the fractions of $\nu_{\mu}$ and $\bar{\nu}_{\mu}$ events that 'disappeared' is $1.9 \sigma$. Extensive checks did not yield any evidence for a bias in the $\bar{\nu}_{\mu}$ event count. When analyzed in terms of the oscillation parameters $\left|\Delta m^{2}\right|$ and $\sin ^{2}(2 \theta)$, the best-fit point for $\nu_{\mu}$ (see Section 2) was found to lie within the $90 \%$ C.L. contour obtained for the $\bar{\nu}_{\mu}$ oscillations.

\section{Conclusions}

All MINOS physics groups are in the process of improving their software, both for data analysis and for simulation, to prepare for processing a new data set representing a beam exposure of $7 \times 10^{20} \mathrm{POT}$, already on hand. The data taking will commence again in October 2009 in a Reverse-Horn-Current Low-Energy beam configuration to provide $\bar{\nu}_{\mu}$ data in the energy range appropriate for the study of antineutrino oscillations.

\section{References}

[1] MINOS Collaboration, D.G. Michael et al., Nucl. Instr. Meth. Phys. Res. A 5961902008

[2] MINOS Collaboration, P. Adamson et al., Phys. Rev. Lett. 101 (2008) 131802

[3] MINOS Collaboration, P. Adamson et al., Phys. Rev. Lett. 101 (2008) 221804 